\title{
Intracerebral Injection of Streptozotocin to Model Alzheimer Disease in Rats
}

Daniel Moreira-Silva1,\#,\$a, Robson C. L. Vizin"1,\#,\$b, Talita M. S. Martins², Tatiana L. Ferreira ${ }^{3, *}$, Maria C. Almeida ${ }^{4, *}$ and Daniel C. Carrettiero, *

${ }^{1}$ Graduate Program in Neuroscience and Cognition, Federal University of $A B C$, São Bernardo do Campo, Brazil; ' ${ }^{2}$ nndergraduate Program in Neuroscience, Federal University of $A B C$, São Bernardo do Campo, Brazil; ${ }^{3}$ Center for Mathematics, Computing and Cognition, Federal University of ABC, São Bernardo do Campo, Brazil; ${ }^{4}$ Center for Natural and Human Sciences, Federal University of ABC, São Bernardo do Campo, Brazil; ${ }^{\$}$ Carrent address: Department of Pharmacology, Institute of Biomedical Sciences, Federal University of Uberlandia, Uberlandia, Brazil; ${ }^{\$ b}$ Current address: Thermoregulation and Systemic Inflammation Laboratory (FeverLab), Trauma Research, St. Joseph's Hospital and Medical Center, Phoenix, EUA

\# Contributed equally to this work

*For correspondence: $\quad \underline{\text { tatiana.ferreira@ufabc.edu.br; } \quad \text { camila.almeida@ufabc.edu.br; }}$ daniel.carrettiero@ufabc.edu.br

[Abstract] Animal models have promoted meaningful contribution to science including Alzheimer's disease $(A D)$ research. Several animal models for $A D$ have been used, most of them related to genetic mutations observed in familial AD. However, sporadic form of $A D$, also named late-onset is the most frequent form of the disease, which is multifactorial, being influenced by genetic, environmental and lifestyle factors. Here, we describe a protocol of an AD-like pathology of the sporadic form using Wistar rats by a single bilateral intracerebroventricular (icv) injection of streptozotocin (STZ, $2 \mathrm{mg} / \mathrm{kg}$ ). Icv injection of STZ induces brain resistance to insulin and other pathological alterations related to those observed in $A D$, such as cognitive impairment and accumulation of phosphorylated tau protein and $\beta$-amyloid in the brain. Thus, icv injection of STZ is a useful tool to investigate the pathological mechanisms and the metabolic alterations involved in $A D$ and to propose new therapeutic approaches and neuroprotective drugs.

Keywords: $\beta$-amyloid, Cognition, Glucose, Insulin, Memory, STZ, Protocol, Tau

[Background] Alzheimer's disease $(A D)$ is the most common cause of senile dementia. One to five percent of $A D$ cases are familial and all the other cases are late-onset also known as sporadic $A D$. The understanding of sporadic $A D$ has progressed substantially from studies using an $A D$ animal model created by intracerebroventricular (icv) injection of streptozotocin (STZ) (Mayer et al., 1990; Lannert and Hoyer, 1998).

STZ is a glucosamine-nitrosourea derived from soil microbe Streptomyces achromogenes. The icv injection of STZ impairs cerebral glucose and energy metabolism, which may be a central event for triggering neurodegeneration in AD (Nitsch and Hoyer, 1991; Plaschke and Hoyer, 1993; Duelli et al., 1994). The mechanisms of icv STZ-induced AD-like pathology were recently reviewed by Grieb (2016) 
and Kamat et al. (2016). The pathological alterations induced by icv injection of STZ are analogous to the features of sporadic $A D$, which include increase in $\beta$-amyloid protein and hyperphosphorylation of tau protein in several brain structures, intensive neuronal death, increase of the brain ventricles, cerebral inflammation, cognitive deficit, anxiety- and depression-like behaviors, decrease in social interaction, increase of core body temperature and cold-avoidance behavior (Kraska et al., 2012; Santos et al., 2012; Chen et al., 2013; Mehla et al., 2013; Knezovic et al., 2015; Dehghan-Shasaltaneh et al., 2016; Crunfli et al., 2018; Moreira-Silva et al., 2018; Motzko-Soares et al., 2018; Vicente et al., 2018; Amani et al., 2019; Crunfli et al., 2019). STZ is usually diluted in saline, citrate buffer, or artificial cerebrospinal fluid, and administrated in a single or multiple injections in a range of $1-3 \mathrm{mg} / \mathrm{kg}$, unilaterally or bilaterally in the lateral ventricles of the brain (Grunblatt et al., 2007; Kraska et al., 2012; Mehla et al., 2013; Dehghan-Shasaltaneh et al., 2016; Adeli et al., 2017). The pathological alteration induced by STZ are progressive and time- and dose-dependent. Thus, icv-STZ injection is characterized by an acute-fast impairment in the memory, followed by partial recovery, and then, a chronic-slow memory impairment (Knezovic et al., 2015). Also, higher doses of STZ promote more severe and fast neurodegeneration and more evident cognitive deficit (Kraska et al., 2012; Knezovic et al., 2015, Dehghan-Shasaltaneh et al., 2016).

Here, we describe a step-by-step protocol used in our laboratory, as summarized in Figure 1. Approximately 30 days from the icv-STZ injection at a dose of $2 \mathrm{mg} / \mathrm{kg}$, clear cognitive impairment and neurodegeneration were observed (Moreira-Silva et al., 2018; Motzko-Soares et al., 2018; Vicente et al., 2018). The protocol used in our laboratory was standardized based on evidences that show: (1) $\mathrm{STZ}$ at high doses ( $\geq 3 \mathrm{mg} / \mathrm{kg}$ ) promotes alteration on locomotor activity (Dehghan-Shasaltaneh et al., 2016), and also induce acute neurotoxic effect with fast severe neurodegeneration that differs from the slow neurodegenerative progress observed in sporadic AD (Kraska et al., 2012); (2) STZ at lower doses ( $\leq 1 \mathrm{mg} / \mathrm{kg}$ ) promotes a very slow neurodegenerative progress and is more appropriate to study neuroprotective drugs (Kraska et al., 2012); (3) an intermediate dose of STZ (around $2 \mathrm{mg} / \mathrm{kg}$ ) was previously reported as the most efficient dose to modeling a sporadic AD-like pathology (Dehghan-Shasaltaneh et al., 2016); (4) multiple injections of STZ increases animal mortality (Mehla et al., 2013); and (5) STZ shows better stability in solution at pH 4.5 than at neutral pH (Junod et al., 1967).

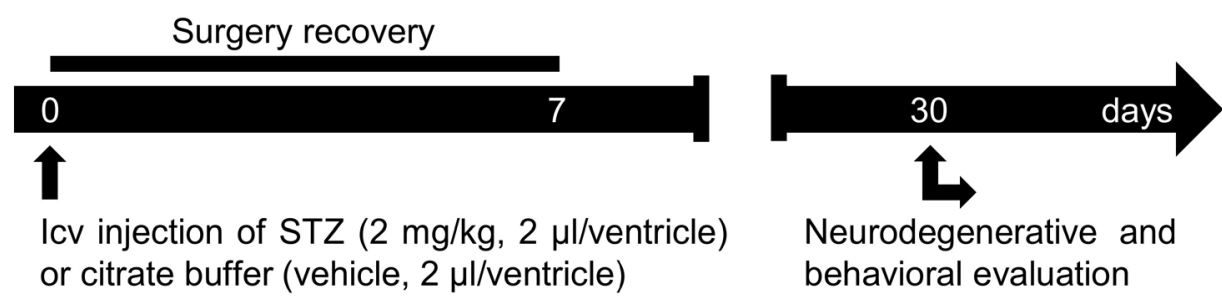

Figure 1. Protocol scheme. STZ (2 mg/kg, $2 \mu \mathrm{l} /$ ventricle) or vehicle (2 $\mu / / v e n t r i c l e)$ is injected in both lateral ventricles of the rat's brain. In the first week post-surgery, animal recovery should be evaluated. After 30 days of icv-STZ injection, animal behavior and the neurodegenerative alterations induced by icv-STZ can be evaluated. 


\section{Materials and Reagents}

\section{A. Animals}

Male Wistar rats (3 to 4 months old, $350-400 \mathrm{~g}$ )

Notes:

a. Institutional animal ethics protocol approval is required to perform the procedure described in this protocol. Personal protective equipment is recommended.

$b$. If the rats are obtained from another source, allow them to habituate to the new colony for at least 3 days before undergoing surgery.

c. In our lab, it was observed that rats under $300 \mathrm{~g}$ of body mass were more susceptible to body mass loss after an icv-STZ injection.

d. Here we recommend male rats since female rats present less sensitivity to STZ-induced cognitive impairment compared to male rats (Bao et al., 2017). Moreover, female rats display differences in some AD typical biomarkers compared to those observed in human. However, these differences were observed in Sprague-Dawley rats. More studies testing the sex differences related to $A D$ susceptibility and progression in other rodent species and strains are needed to better understand how the hormonal modulation could affect the disease progression. As $A D$ is more prevalent between women than men, the fact that STZ models are generally applied only to male rodents is a bias that restricts the representativeness of the model (Bao et al., 2017).

B. STZ and vehicle preparation

1. Spatula (Sigma-Aldrich, catalog number: Z648299)

2. Beaker $50 \mathrm{ml}$ (Qualividros, catalog number: 59930)

3. Pipette tips 0.1-10 $\mu \mathrm{l}$ (VWR, catalog number: 53509-138)

4. Microcentrifuge tube $0.5 \mathrm{ml}$ (Eppendorf, catalog number: 0030124537)

5. STZ (Santa Cruz Biotechnology, catalog number: sc-200719)

6. Sodium citrate (Sigma-Aldrich, catalog number: W302600)

7. Sodium chloride (Sigma-Aldrich, catalog number: S7653)

8. Hydrochloric acid (Sigma-Aldrich, catalog number: 320331)

9. Sodium hydroxide (Sigma-Aldrich, catalog number: S8045)

10. Double distilled water

11. $0.05 \mathrm{M}$ Citrate buffer (see Recipes)

C. Surgery

1. Aluminum foil

2. Scissors (Roboz, catalog number: RS-6700)

3. Tissue forceps (Roboz, catalog number: RS-8166 or RS-5242)

4. Suture Needle (Aspen Surgical, Richard-Allan ${ }^{\circledR}$ needle, catalog number: 200016) 
5. Suture thread (Braunamid, catalog number: F1185047)

6. Cotton gauze (Fisherbrand, catalog number: 22-362178)

7. Micropipette (Gilson Inc., catalog number: F81022)

8. $1 \mathrm{ml}$ syringe (BD, catalog number: 309628$)$

9. $22 \mathrm{G}$ needle (BD, catalog number: 305159$)$

10. $25 \mathrm{G}$ needle (BD, catalog number: 305122)

11. $24 \mathrm{G}$ intravenous catheter (Terumo, catalog number: SR-OX2419CA)

12. Polyethylene tubing PE-10 (Scientific Commodities Inc., catalog number: BB31695-PE/1)

13. Microsyringe $10 \mu \mathrm{l}$ (Hamilton, catalog number: 80300)

14. Ketamine (Syntec)

15. Xylazine (Syntec)

16. (Optional) Lidocaine and epinephrine

17. Pentabiotic (Zoetis)

18. Ketoprofen (Ceva)

19. Sterile water for injection (Equiplex)

20. Povidone-iodine alcoholic solution (Aurora Pharmaceutical, catalog number: 19004)

\section{Equipment}

1. Molt elevator (Roboz, catalog number: RS-8830)

2. Needle holder (Fine Science Tools, catalog number: 12010-14)

3. Analytical scale (Marte, model: ATX 224)

4. $\mathrm{pH}$ meter (Marte, model: MB10)

5. Small animal scale (Bonther, model: LS5)

6. Pet hair clipper (Amplavet, catalog number: 950042)

7. Stereotaxic apparatus (Kopf, model: 900)

8. Heating pad (PhysioSuite ${ }^{\circledR}$, Kent Scientific, catalog number: PS-02)

9. Dental drill (Bonther, model: BL 2000)

10. Infusion pump (Insight Equipment, model: Bi2000, catalog number: EFF 311)

11. $-20{ }^{\circ} \mathrm{C}$ freezer (VWR, model: SCBMF-2020)

12. Autoclave (Prismatec, model: CS)

\section{Software}

1. Sigma Plot 11 or Statistica 7.0

\section{Procedure}

A. Surgery setup 
1. Sterilize all the surgical tools.

2. Prepare the citrate buffer as described in "Recipes".

3. In a dedicated surgery room, place all the materials and equipment to be used in the surgery.

4. Measure and take note of the body mass of each rat.

5. In microcentrifuge tubes, individually weigh the amount of STZ needed for each rat based on its body mass. For example, for a $350 \mathrm{~g}$ rat, weigh $1.05 \mathrm{mg}$ of STZ which later will be diluted in $6 \mu \mathrm{l}$ of citrate buffer. Although only a total of $4 \mu \mathrm{l}$ of this solution will be injected $(2 \mu \mathrm{l}$ per lateral ventricle), which will correspond to a dose of $2 \mathrm{mg} / \mathrm{kg}$, we recommended preparing a larger volume of solution.

Note: STZ is health hazard and personal protective equipment is required. The STZ solution has a very short half-life (15-30 $\mathrm{min})$ and is unstable. Solutions should be prepared fresh before use.

6. Cover the microcentrifuge tubes with aluminum foil to protect STZ from light.

7. Number each microcentrifuge tube with STZ with the corresponding rat's identification number.

8. Keep the microcentrifuge tube with $\mathrm{STZ}$ at $-20^{\circ} \mathrm{C}$.

9. Prepare the microinjection system. For this, cut the $24 \mathrm{G}$ intravenous catheter using a drill to make a $15 \mathrm{~mm}$ longer STZ-injection needle. Connect the STZ-injection needle to the end of the PE-10 tubing (40 cm longer). Fill the PE-10 tubing with water for injection and connect the other end to a $10 \mu \mathrm{l}$ Microsyringe coupled to an infusion pump.

Note: Always make sure the microinjection system is not clogged. Set the infusion pump to run at $1 \mu \mathrm{l} / \mathrm{min}$ rate.

B. Icv injection of STZ (see Figures 2 and 3)

Note: Surgery should be performed under sterile conditions. We recommend lubricating the eyes of the rat with saline or eye drops during surgery.

1. Anesthetize the rat with an association of ketamine $(90 \mathrm{mg} / \mathrm{kg})$ and xylazine $(10 \mathrm{mg} / \mathrm{kg})$, both administered intraperitoneally using $25 \mathrm{G}$ needles and $1 \mathrm{ml}$ syringe.

Note: It is important to assure that the rat is completely anesthetized and not responsive to a possible painful stimulus. You can check the responsiveness of the rat by pressing the tip of its tail or the sole of its posterior paws. If the rat presents any reaction after $5 \mathrm{~min}$ of the injection, the ketamine has to be reinforced up to $50 \%$ of the initial dose.

2. Place the rat in the stereotaxic apparatus over the heating pad and maintain rectal temperature around $37^{\circ} \mathrm{C}$.

3. Administer the pentabiotic $(48,000 \mathrm{UI}$, intramuscular) and the ketoprofen $(5 \mathrm{mg} / \mathrm{kg}$, subcutaneous), using the $22 \mathrm{G}$ and $25 \mathrm{G}$ needles, respectively, and $1 \mathrm{ml}$ syringe.

4. Shear the dorsal region of the head and clean the area designated for the procedure with the povidone-iodine alcoholic solution.

Note: Be very careful not to shave the vibrissae.

5. Using the tissue forceps and the scissors, incise the scalp over the sagittal suture 
(approximately $2 \mathrm{~cm}$ long) and excise the periosteum to expose the skull. Use the Molt elevator to help in this process.

Note: Optionally, local anesthetic consisting of 2\% lidocaine and epinephrine (1: 200,000) can be injected subcutaneously approximately 1 min before the incision to reduce bleeding.

6. Stereotaxic icv-injections are made with bregma and lambda on the same horizontal plane. For this, check the dorsoventral axis of lambda and bregma. A difference in the lambda and bregma dorsoventral axis under $0.2 \mathrm{~mm}$ indicates a correct position of the rat's head.

7. Drill two small holes in the skull, just large enough to allow the STZ-injection needle to cross through it and penetrate the brain surface, following the coordinates measured from the bregma, to reach the lateral ventricles: $-0.8 \mathrm{~mm}$ in the anteroposterior axis, $\pm 1.4 \mathrm{~mm}$ in the mediolateral axis and $-3.6 \mathrm{~mm}$ in the dorsoventral axis (Paxinos et al., 2005).

8. Using a micropipette, add the citrate buffer to the STZ previously weighted in a microcentrifuge tube and homogenize it drawing the solution in and dispensing it in the microcentrifuge tube a couple of times.

Note: It is expected that the STZ solution presents a slightly yellow color and few bubbles can be observed due to NO release. STZ solutions should be prepared fresh before use.

9. Aspirate $1 \mu \mathrm{l}$ of air and then aspirate the STZ solution using the STZ-injection needle previously placed in the arm of the stereotaxic device, which is connected to an automated microinjection system. The $1 \mu \mathrm{l}$ of air is to create a bubble between the STZ solution and the water that is filling the PE-10 tubing.

10. Lower slowly the STZ-injection needle inside the rat brain, passing through one of the skull holes, following the lateral ventricle coordinates.

11. Initiate the icv-STZ injection by activating the infusion pump at a rate of $1 \mu \mathrm{l} / \mathrm{min}$ for $2 \mathrm{~min}$. Thus, $2 \mu \mathrm{l}$ of STZ solution should be injected in each lateral ventricle.

Note: Monitor the flow of the injection through the bubble path along the PE-10 tube.

12. At the end of injection, wait 2 min to withdraw the STZ-injection needle from the lateral ventricle of the brain in order to prevent the reflux of the STZ solution.

13. Repeat the same procedure to the other lateral ventricle of the brain.

14. At the end of STZ injection in both lateral ventricles of the brain, suture the skin.

15. For control rats, inject $2 \mu \mathrm{l}$ of citrate buffer, instead of the STZ, in each lateral ventricle of the brain, exactly as described in Steps B9-B14.

Note: For citrate buffer injection, use a different injection needle and PE-10 tubing from that used for STZ injection.

16. Administer $3 \mathrm{ml}$ of saline subcutaneously to rehydrate the rat.

17. Allow the rat to recovery in an individual cage for at least 3 days.

Note: The rat should be under constant observation until its recovery from anesthesia (approximately one hour) and for the following first-week post-surgery. Physiological parameters should be evaluated daily, such as body mass, food, and water intake, as well as behaviors associated with pain and discomfort. If necessary, supplementary feeding, and 
additional analgesic, anti-inflammatory, and antibiotic medication can be administered under the recommendation of the veterinarian in charge. Rats suffering irreversible conditions of pain and weight loss should be euthanized. ICV-STZ injected rats may have more difficult to recover from the surgery compared to control rats.

18. Return the rat to the colony.

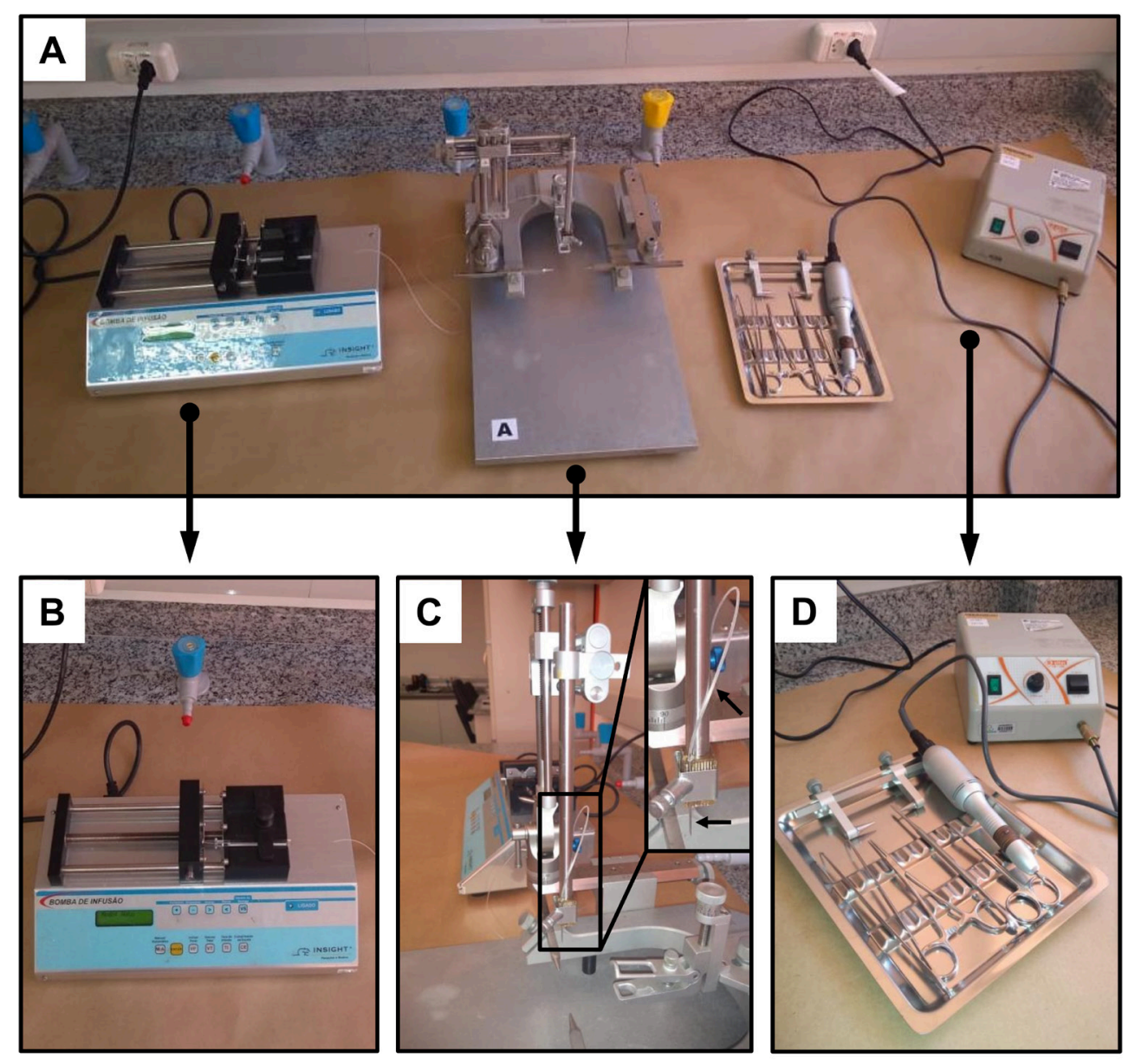

Figure 2. Setup for the injection procedure. A. Main equipment and tools used for the STZ-injection surgery. B. Microinjection system: infusion pump with a microsyringe connected to one end of a PE-10 tubing. C. Stereotaxic apparatus (lateral view) with the STZ-injection needle connected to another end of the PE-10 tubing, as indicated by arrows. D. Surgical tools and dental drill.

C. Evaluation of the cognitive performance of the rats

Note: As $A D$ is characterized in human mainly for their multiple cognitive deficits, to guarantee that the $A D$ animal model mimics most of the aspects of the disease, cognitive testing is essential to detect memory deficits of the animals. For the STZ-model described here, we recommend that behavioral tests be carried out only after surgery full recovery, when animals reach similar body weight values compared to the day before the surgery and present a completely healthy state (approximately one week after surgery). 
Depending on the cognitive tests used, early deficits can be observed but most of the studies generally perform the behavioral and histopathological analysis one month after surgery, when most of the mimicked aspects of $A D$ can be observed, such as the accumulation of phosphorylated tau protein and ventricle enlargement (Knezovic et al., 2015; Moreira-Silva et al., 2018). In this AD model, several types of memory deficits can be evaluated by using the following tasks: the Barnes' maze, object displacement and the Morris' water maze for spatial memory; radial and Y-maze for working memory; inhibitory avoidance, escape latency in the elevated plus maze and fear conditioning for emotional memory and new object recognition for recognition memory. The full description of some behavioral tasks can be found in previous studies published by our group (Moreira-Silva et al., 2018, for new object recognition, fear conditioning and escape latency in the elevated plus maze and Motzko-Soares et al., 2018, for Barnes' and Y-maze). Also, histopathological and molecular analyses of the presence of AD biomarkers induced by icv-STZ injection were described previously by our research group (Moreira-Silva et al., 2018, Motzko-Soares et al., 2018).
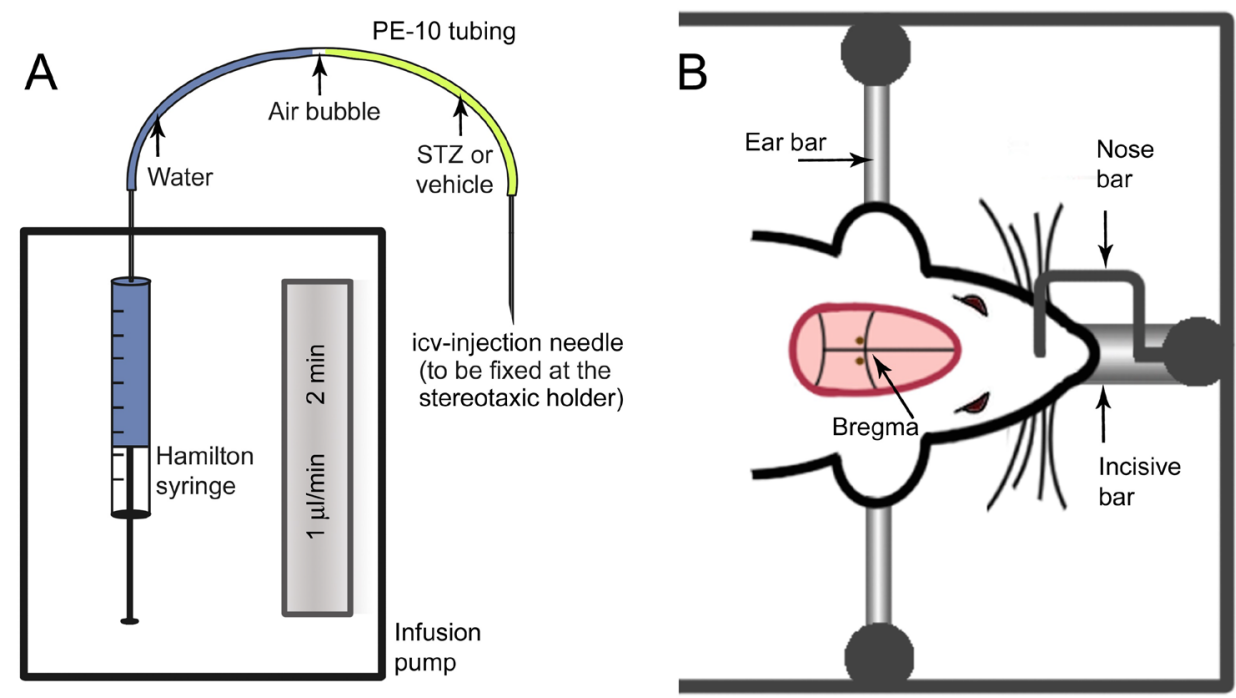

Figure 3. Schematics of the setup for the injection procedure (A) and top view of the rats' head in the stereotaxic apparatus (B). In B, the reddish indicates the sheared area in the rats' head. The two brown points posterior to the bregma indicates the approximate position of the holes drilled for the insertion of the icv injection needle.

\section{Data analysis}

In general, behavioral tests request a large experimental number of animals. We suggest an experimental number of $>10$ rats per group. The data from our laboratory have shown a normal distribution and were evaluated by parametrical tests such as Student's $t$-test, and One- or Two-way ANOVA using Sigma Plot 11 or Statistica 7.0 software. A detailed description of data analysis and experimental number for each group and test can be found at "Data processing and 
analysis/Statistical Analysis" section, and in the legends of the figures, respectively, in Moreira-Silva et al. (2018), Motzko-Soares et al. (2018), and Vicente et al. (2018). Figure 4 shows the behavioral (A) and histopathological (B-D) alterations induced by icv injection of STZ $(2 \mathrm{mg} / \mathrm{kg})$, as examples of tests to be performed post-surgery. Increase in $\beta$-amyloid protein expression and hyperphosphorylation of Tau is observed in several brain structures of icv-STZ-injected rats (Santos, et al., 2012; Knezovic et al., 2015; Dehghan-Shasaltaneh et al., 2016).

A

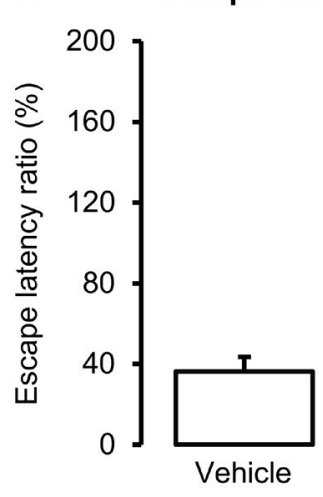

B

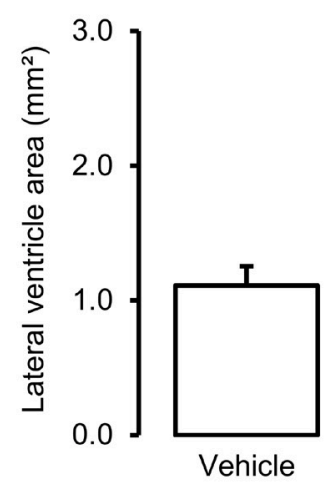

C Vehicle

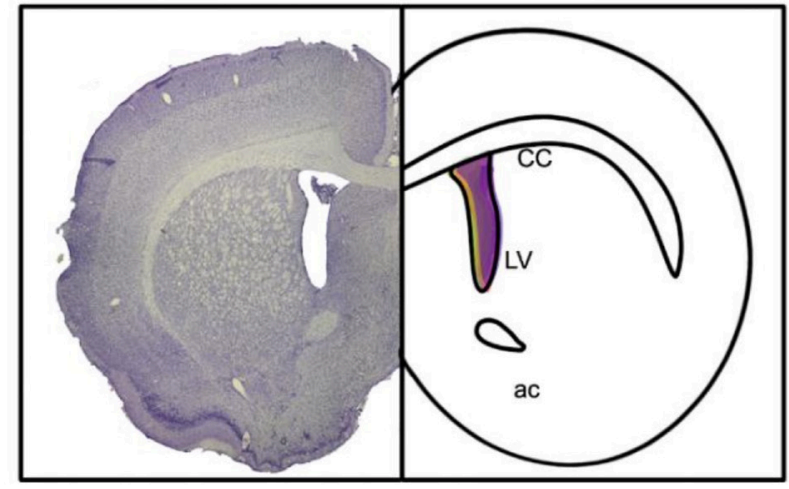

D

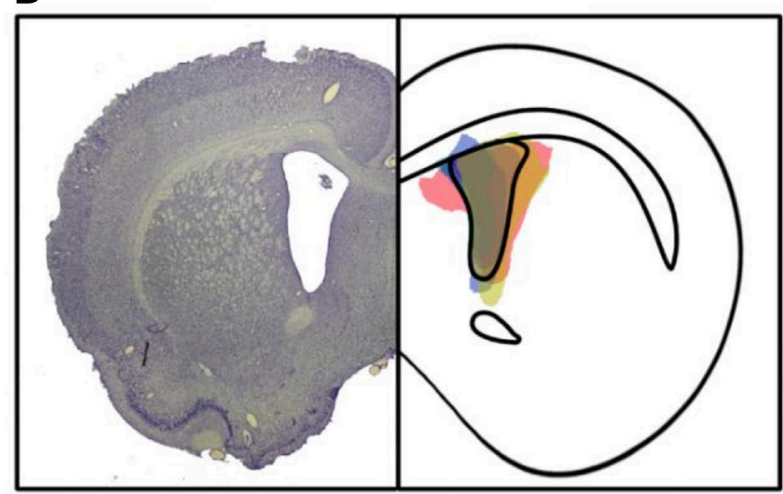

Figure 4. Behavioral and histopathological analyses of Wistar rats submitted to the icv injection of STZ $(2 \mathbf{~ m g} / \mathbf{k g})$. A. Effect of icv-STZ injection in the Escape latency test evaluated in the elevated-plus maze (EPM). Escape latency is increased in STZ-treated rats 30 days after the surgery compared to vehicle-treated rats, indicating impairment in emotional memory. Escape latency was calculated by the equation: (time spent to find the closed arm in the test/ time spent to find the closed arm in the training) $\times 100$. For the escape latency test protocol, see the original publication. Data are represented as mean $\pm S E(n=11-12$ rats per group). $B$. Ventricle area $\left(\mathrm{mm}^{2}\right)$ of STZ- and vehicle-treated rats measured $1.56 \mathrm{~mm}$ posterior to bregma, 40 days after the surgery. The icv-STZ injection increased the lateral ventricle area compared to vehicle-treated rats, indicating neurodegeneration. Data are represented as mean $\pm S E$. C and $D$. Showing representative histological images of the lateral ventricles of the vehicle and icv-STZ groups. The right panel represents schematically the shape of the histological figure from the left, filled with colored spots corresponding to the ventricular area of each sample 
used in the respective groups ( $\mathrm{n}=3-5$ per group). ${ }^{*}$ indicate $P \leq 0.05$ (Student's $t$-test). LV: lateral ventricle; CC: Corpus callosum. A-D are adapted from Moreira-Silva et al. (2018).

\section{Recipes}

1. $0.05 \mathrm{M}$ citrate buffer
a. Transfer $40 \mathrm{ml}$ of distilled water in a beaker
b. Add $0.735 \mathrm{~g}$ of Sodium Citrate (molar weight: $294.1 \mathrm{~g} / \mathrm{mol}$ ) to the solution
c. Add $0.450 \mathrm{~g}$ of Sodium chloride (molar weight: $58.4 \mathrm{~g} / \mathrm{mol}$ ) to the solution
d. Adjust the solution to a final $\mathrm{pH}$ of 4.5 using $\mathrm{HCl}$ or $\mathrm{NaOH}$
e. Add distilled water to adjust the volume to $50 \mathrm{ml}$
f. Autoclave the solution and keep it at $4{ }^{\circ} \mathrm{C}$

Note: Use the citrate buffer solution at room temperature.

\section{Acknowledgments}

The authors thank the financial support by São Paulo Research Foundation-FAPESP (undergraduate fellowship grant 2017/12648-6 to T.M.S.M, graduate fellowship grants 2014/14661-1 and 2016/24773-7 to D.M-S, and 201325503-5 and 201601836-3 to R.C.L.V.; research grants 2015/02991-0 to M.C.A. and 2015/23426-9 to D.C.C.), CNPq (research grant 429894/2016-3 to T.L.F. and 449102/2014-9 to D.C.C.), CNPq (grants 429894/2016-3; 449102/2014-9), and Federal University of ABC (UFABC).

\section{Competing interests}

The authors declare no financial and non-financial competing interests.

\section{Ethics}

All procedures were approved by the Committee on Ethics in Animal Use of the Federal University of $A B C$ (CEUA-UFABC), protocol 008/13.

\section{References}

1. Adeli, S., Zahmatkesh, M., Tavoosidana, G., Karimian, M. and Hassanzadeh, G. (2017). Simvastatin enhances the hippocampal klotho in a rat model of streptozotocin-induced cognitive decline. Prog Neuropsychopharmacol Biol Psychiatry 72: 87-94.

2. Amani, M., Zolghadrnasab, M. and Salari, A. A. (2019). NMDA receptor in the hippocampus alters neurobehavioral phenotypes through inflammatory cytokines in rats with sporadic 
Alzheimer-like disease. Physiol Behav 202: 52-61.

3. Bao, J., Mahaman, Y. A. R., Liu, R., Wang, J. Z., Zhang, Z., Zhang, B. and Wang, X. (2017). Sex differences in the cognitive and hippocampal effects of streptozotocin in an animal model of sporadic AD. Front Aging Neurosci 9: 347.

4. Chen, Y., Liang, Z., Blanchard, J., Dai, C. L., Sun, S., Lee, M. H., Grundke-lqbal, I., Iqbal, K., Liu, F. and Gong, C. X. (2013). A non-transgenic mouse model (icv-STZ mouse) of Alzheimer's disease: similarities to and differences from the transgenic model (3xTg-AD mouse). Mol Neurobiol 47(2): 711-725.

5. Crunfli, F., Mazucanti, C. H., de Moraes, R. C. M., Costa, A. P., Rodrigues, A. C., Scavone, C. and Torrao, A. S. (2018). NO-dependent Akt inactivation by S-Nitrosylation as a possible mechanism of STZ-Induced neuronal insulin resistance. J Alzheimers Dis 65(4): 1427-1443.

6. Crunfli, F., Vrechi, T. A., Costa, A. P. and Torrao, A. S. (2019). Cannabinoid receptor type 1 agonist ACEA improves cognitive deficit on STZ-Induced neurotoxicity through apoptosis pathway and NO modulation. Neurotox Res 35(3): 516-529.

7. Dehghan-Shasaltaneh, M., Naghdi, N., Choopani, S., Alizadeh, L., Bolouri, B., Masoudi-Nejad, A. and Riazi, G. H. (2016). Determination of the best concentration of streptozotocin to create a diabetic brain using histological techniques. J Mol Neurosci 59(1): 24-35.

8. Duelli, R., Schrock, H., Kuschinsky, W. and Hoyer, S. (1994). Intracerebroventricular injection of streptozotocin induces discrete local changes in cerebral glucose utilization in rats. Int J Dev Neurosci 12(8): 737-743.

9. Grieb, P. (2016). Intracerebroventricular streptozotocin injections as a model of Alzheimer's disease: in search of a relevant mechanism. Mol Neurobiol 53(3): 1741-1752.

10. Grunblatt, E., Salkovic-Petrisic, M., Osmanovic, J., Riederer, P. and Hoyer, S. (2007). Brain insulin system dysfunction in streptozotocin intracerebroventricularly treated rats generates hyperphosphorylated tau protein. $J$ Neurochem 101(3): 757-770.

11. Junod, A., Lambert, A. E., Orci, L., Pictet, R., Gonet, A. E. and Renold, A. E. (1967). Studies of the diabetogenic action of streptozotocin. Proc Soc Exp Biol Med 126(1): 201-205.

12. Kamat, P. K., Kalani, A., Rai, S., Tota, S. K., Kumar, A. and Ahmad, A. S. (2016). Streptozotocin intracerebroventricular-induced neurotoxicity and brain insulin resistance: a therapeutic intervention for treatment of sporadic Alzheimer's disease (SAD)-like pathology. Mol Neurobiol 53(7): 4548-4562.

13. Knezovic, A., Osmanovic-Barilar, J., Curlin, M., Hof, P. R., Simic, G., Riederer, P. and Salkovic-Petrisic, M. (2015). Staging of cognitive deficits and neuropathological and ultrastructural changes in streptozotocin-induced rat model of Alzheimer's disease. J Neural Transm (Vienna) 122(4): 577-592.

14. Kraska, A., Santin, M. D., Dorieux, O., Joseph-Mathurin, N., Bourrin, E., Petit, F., Jan, C., Chaigneau, M., Hantraye, P., Lestage, P. and Dhenain, M. (2012). In vivo cross-sectional characterization of cerebral alterations induced by intracerebroventricular administration of streptozotocin. PLoS One 7(9): e46196. 
15. Lannert, H. and Hoyer, S. (1998). Intracerebroventricular administration of streptozotocin causes long-term diminutions in learning and memory abilities and in cerebral energy metabolism in adult rats. Behav Neurosci 112(5): 1199-1208.

16. Mayer, G., Nitsch, R. and Hoyer, S. (1990). Effects of changes in peripheral and cerebral glucose metabolism on locomotor activity, learning and memory in adult male rats. Brain Res 532(1-2): 95-100.

17. Mehla, J., Pahuja, M. and Gupta, Y. K. (2013). Streptozotocin-induced sporadic Alzheimer's disease: selection of appropriate dose. J Alzheimers Dis 33(1): 17-21.

18. Moreira-Silva, D., Carrettiero, D. C., Oliveira, A. S. A., Rodrigues, S., Dos Santos-Lopes, J., Canas, P. M., Cunha, R. A., Almeida, M. C. and Ferreira, T. L. (2018). Anandamide effects in a streptozotocin-induced Alzheimer's disease-like sporadic dementia in rats. Front Neurosci 12: 653.

19. Motzko-Soares, A. C. P., Vizin, R. C. L., Martins, T. M. S., Hungaro, A. R. O., Sato, J. R., Almeida, M. C. and Carrettiero, D. C. (2018). Thermoregulatory profile of neurodegeneration-induced dementia of the Alzheimer's type using intracerebroventricular streptozotocin in rats. Acta Physiol (Oxf) 224(2): e13084.

20. Nitsch, R. and Hoyer, S. (1991). Local action of the diabetogenic drug, streptozotocin, on glucose and energy metabolism in rat brain cortex. Neurosci Lett 128(2): 199-202.

21. Paxinos, G. and Watson, C. (2005) The rat brain in stereotaxic coordinates. 5th Ed. San Diego, Elsevier Academic Press.

22. Plaschke, K. and Hoyer, S. (1993). Action of the diabetogenic drug streptozotocin on glycolytic and glycogenolytic metabolism in adult rat brain cortex and hippocampus. Int J Dev Neurosci 11(4): 477-483.

23. Santos, T. O., Mazucanti, C. H., Xavier, G. F. and Torrao, A. S. (2012). Early and late neurodegeneration and memory disruption after intracerebroventricular streptozotocin. Physiol Behav 107(3): 401-413.

24. Vicente, M. C., Almeida, M. C., Bicego, K. C., Carrettiero, D. C. and Gargaglioni, L. H. (2018). Hypercapnic and hypoxic respiratory response during wakefulness and sleep in a streptozotocin model of Alzheimer's disease in rats. J Alzheimers Dis 65(4): 1159-1174. 